<smiles>Cc1ccc(/N=C/c2ccc(/C=N/c3ccc(C)cc3)s2)cc1</smiles>

\section{Crystal structure of $N, N^{\prime}$-[(thiophene- 2,5-diyl)bis(methanylylidene)]di-p-tolui- dine}

\section{Raina Boyle, Guy Crundwell* and Neil M. Glagovich}

Department of Chemistry \& Biochemistry, Central Connecticut State University, New Britain, CT 06053, USA. *Correspondence e-mail: crundwellg@mail.ccsu.edu

Received 11 April 2015; accepted 29 April 2015

Edited by A. J. Lough, University of Toronto, Canada

The title compound, $\mathrm{C}_{20} \mathrm{H}_{18} \mathrm{~N}_{2} \mathrm{~S}$, was synthesized by the condensation reaction between $p$-toluidine and thiophene-2,5dicarboxaldehye in refluxing toluene with $p$-toluenesulfonic acid added as catalyst. The molecule lies on a twofold rotation axis and adopts an $E$ orientation with respect to the azomethine bonds. The dihedral angle between the unqiue benzene ring and the least-squares plane [maximum deviation $=0.0145(14) \AA]$ containing the azomethine and thiophene groups is $32.31(6)^{\circ}$.

Keywords: crystal structure; symmetrical diazomethine.

CCDC reference: 1062484

\section{Related literature}

For the synthesis of the title compound, see: Vaysse \& Pastour (1964). For the syntheses and crystal structures of molecules related to the title compound, see: Bernès et al. (2013); Mendoza et al. (2014). For applications of symmetrical diazomethines, see: Suganya et al. (2014); Skene \& Dufresne (2006). For related structures, see: Bolduc et al. (2013).

\section{Experimental}

2.1. Crystal data
$\mathrm{C}_{20} \mathrm{H}_{18} \mathrm{~N}_{2} \mathrm{~S}$

$M_{r}=318.42$

Monoclinic, $C 2 / c$

$a=37.166(2) \AA$

$b=6.0292(2) \AA$

$c=7.5814(4) \AA$

$\beta=93.452(7)^{\circ}$

\subsection{Data collection}

Oxford Diffraction Xcalibur

Absorption correction: multi-scan (CrysAlis PRO; Oxford Diffraction, 2009)

$T_{\min }=0.713, T_{\max }=1.000$

\subsection{Refinement}

$R\left[F^{2}>2 \sigma\left(F^{2}\right)\right]=0.048$

$w R\left(F^{2}\right)=0.145$

$S=1.03$

2861 reflections Sapphire3 diffractometer

$$
\begin{aligned}
& V=1695.78(15) \AA^{3} \\
& Z=4 \\
& \text { Mo } K \alpha \text { radiation } \\
& \mu=0.19 \mathrm{~mm}^{-1} \\
& T=298 \mathrm{~K} \\
& 0.32 \times 0.24 \times 0.07 \mathrm{~mm}
\end{aligned}
$$

9577 measured reflections 2153 reflections with $I>2 \sigma(I)$ $R_{\text {int }}=0.044$ 2861 independent reflections

Data collection: CrysAlis CCD (Oxford Diffraction, 2009); cell refinement: CrysAlis RED (Oxford Diffraction, 2009); data reduction: CrysAlis RED; $\operatorname{program}(\mathrm{s})$ used to solve structure: SHELXS2014 (Sheldrick, 2008); program(s) used to refine structure: SHELXL2014 (Sheldrick, 2015); molecular graphics: ORTEP-3 for Windows (Farrugia, 2012); software used to prepare material for publication: SHELXL2014 (Sheldrick, 2015).

\section{Acknowledgements}

This research was funded by a CCSU-AAUP research grant.

Supporting information for this paper is available from the IUCr electronic archives (Reference: LH5761).

\section{References}

Bernès, S., Hernández-Téllez, G., Sharma, M., Portillo-Moreno, O. \& Gutiérrez, R. (2013). Acta Cryst. E69, o1428.

Bolduc, A., Dufresne, S. \& Skene, W. G. (2013). Acta Cryst. C69, 1196-1199. Farrugia, L. J. (2012). J. Appl. Cryst. 45, 849-854.

Mendoza, A., Bernès, S., Hernández-Téllez, G., Portillo-Moreno, O. \& Gutiérrez, R. (2014). Acta Cryst. E70, o345.

Oxford Diffraction (2009). CrysAlis CCD, CrysAlis PRO and CrysAlis RED. Oxford Diffraction Ltd, Abington, Oxfordshire, England.

Sheldrick, G. M. (2008). Acta Cryst. A64, 112-122.

Sheldrick, G. M. (2015). Acta Cryst. C71, 3-8.

Skene, W. G. \& Dufresne, S. (2006). Acta Cryst. E62, o1116-o1117.

Suganya, S., Velmathi, S. \& MubarakAli, D. (2014). Dyes Pigments, 104, 116122. 


\section{supporting information}

Acta Cryst. (2015). E71, o403 [doi:10.1107/S205698901500849X]

\section{Crystal structure of $N, N^{\prime}$-[(thiophene-2,5-diyl)bis(methanylylidene)]di-p- toluidine}

\section{Raina Boyle, Guy Crundwell and Neil M. Glagovich}

\section{S1. Comment}

Schiff base condensation reactions between between aldehydes and amines are commonplace in the chemical literature due to the ease of synthesis, isolation, and purification. The title compound was first synthesized by Vaysse \& Pastour in 1964. Recent structural studies of symmetrical diazomethines have appeared in this journal and others due to interests in solvent-free reactions (Bernès, et al. 2013; Mendoza, et al. 2014), in cation sensors (Suganya, et al. 2014) and in photoactive materials (Skene \& Dufresne, 2006).

The molecular structure of the title compound is shown in Fig. 1. The molecule lies on a twofold rotation axis thereby having exact $\mathrm{C}_{2}$ molecular symmetry. The molecule adopts an $E$ orientation with respect to the azomethine bonds. The dihedral angle between the benzene ring (C4-C9) and the least-squares plane (with maximum deviaton 0.0145 (14) $\AA$ for $\mathrm{C} 3)$ containing the azomethine and thiophene groups $\left(\mathrm{S} 1 / \mathrm{C} 1 / \mathrm{C} 2 / \mathrm{C} 1^{i} \mathrm{C} 2^{i} / \mathrm{N} 1 / \mathrm{C} 3\right.$; symmetry code: (i) $\left.-\mathrm{x}+2, \mathrm{y},-\mathrm{z}+3 / 2\right)$ is $32.31(6)^{\circ}$. The crystal structures of some related symmetrical azomethine compounds appear in the literature (Bolduc et al., 2013).

\section{S2. Experimental}

To a $100 \mathrm{ml}$ round-bottomed flask equipped with a Dean-Stark trap and a reflux condenser were added $p$-toluidine (1.77 $\mathrm{g}, 16.5 \mathrm{mmol}), 2,5$-thiophenecarboxaldehye $(0.7602 \mathrm{~g}, 5.4 \mathrm{mmol}), p$-toluenesulfonic acid $(0.0010 \mathrm{~g}, 0.54 \mathrm{mmol})$ and toluene $(50 \mathrm{ml})$ in a method similar to Suganya, et al., 2014). The resulting mixture was refluxed for $24 \mathrm{~h}$ and the yellow solution was concentrated open to the air, producing a yellow solid. The synthesis of the title compound was also accomplished using solvent-free direct grinding method (Bernès, et al. 2013; Mendoza, et al. 2014). The solid was purified by recrystallization in an equal volume mix of toluene and methanol. Crystals were grown from a $p$-xylene solution.

\section{S3. Refinement}

Hydrogen atoms on $s p^{2}$ atoms were included in calculated positions with a $\mathrm{C}-\mathrm{H}$ distance of $0.93 \AA$ and were included in the refinement in riding motion approximation with $U_{\text {iso }}=1.2 U_{\text {eq }}$ of the carrier atom.

Hydrogen atoms on $s p^{3}$ atoms were included in calculated positions with a $\mathrm{C}-\mathrm{H}$ distance of $0.98 \AA$ and were included in the refinement in riding motion approximation with $U_{\text {iso }}=1.5 U_{\text {eq }}$ of the carrier atom. 


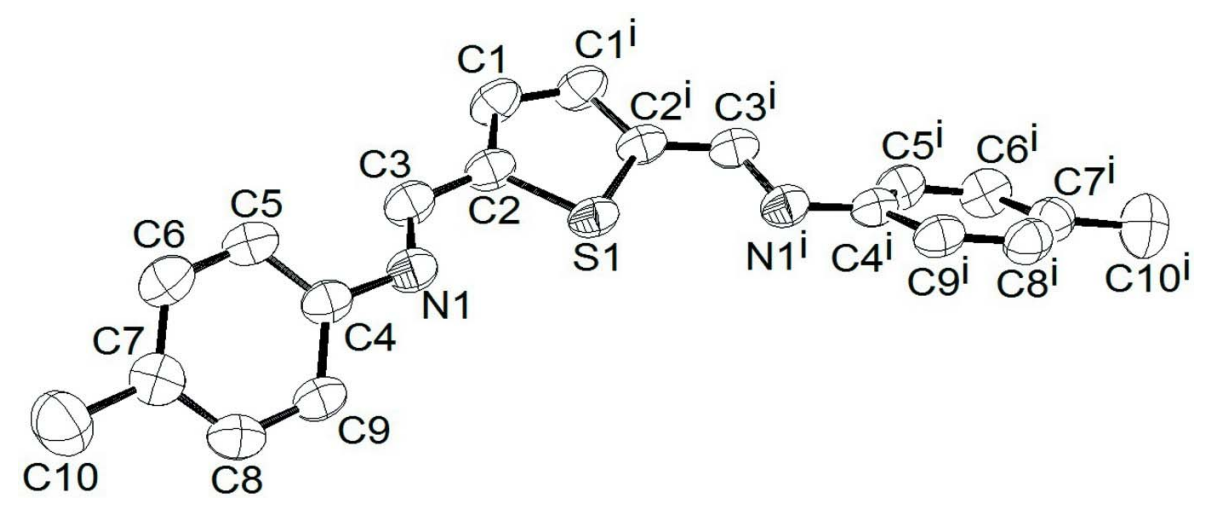

\section{Figure 1}

A view of the title compound (Farrugia, 2012). Displacement ellipsoids are drawn at the $50 \%$ probability level [symmetry code: (i) $-x+2, y,-z+3 / 2]$.

\section{$N, N^{\prime}$-[(Thiophene-2,5-diyl)bis(methanylylidene)]di-p-toluidine}

Crystal data

\section{$\mathrm{C}_{20} \mathrm{H}_{18} \mathrm{~N}_{2} \mathrm{~S}$}

$M_{r}=318.42$

Monoclinic, $C 2 / c$

$a=37.166(2) \AA$

$b=6.0292(2) \AA$

$c=7.5814(4) \AA$

$\beta=93.452(7)^{\circ}$

$V=1695.78(15) \AA^{3}$

$Z=4$

$F(000)=672$

\section{Data collection}

Oxford Diffraction Xcalibur Sapphire3 diffractometer

Radiation source: Enhance (Mo) X-ray Source Graphite monochromator

Detector resolution: 16.1790 pixels $\mathrm{mm}^{-1}$

$\omega$ scans

Absorption correction: multi-scan

(CrysAlis PRO; Oxford Diffraction, 2009)

$T_{\min }=0.713, T_{\max }=1.000$

\section{Refinement}

Refinement on $F^{2}$

Least-squares matrix: full

$R\left[F^{2}>2 \sigma\left(F^{2}\right)\right]=0.048$

$w R\left(F^{2}\right)=0.145$

$S=1.03$

2861 reflections

106 parameters

0 restraints
$D_{\mathrm{x}}=1.247 \mathrm{Mg} \mathrm{m}^{-3}$

Melting point: $508 \mathrm{~K}$

Mo $K \alpha$ radiation, $\lambda=0.71073 \AA$

Cell parameters from 5038 reflections

$\theta=4.3-32.6^{\circ}$

$\mu=0.19 \mathrm{~mm}^{-1}$

$T=298 \mathrm{~K}$

Plate, yellow

$0.32 \times 0.24 \times 0.07 \mathrm{~mm}$

9577 measured reflections

2861 independent reflections

2153 reflections with $I>2 \sigma(I)$

$R_{\text {int }}=0.044$

$\theta_{\text {max }}=32.6^{\circ}, \theta_{\min }=4.3^{\circ}$

$h=-55 \rightarrow 44$

$k=-8 \rightarrow 9$

$l=-10 \rightarrow 10$

Hydrogen site location: inferred from neighbouring sites

$\mathrm{H}$-atom parameters constrained

$w=1 /\left[\sigma^{2}\left(F_{\mathrm{o}}^{2}\right)+(0.0795 P)^{2}+0.2248 P\right]$

where $P=\left(F_{\mathrm{o}}{ }^{2}+2 F_{\mathrm{c}}{ }^{2}\right) / 3$

$(\Delta / \sigma)_{\max }=0.001$

$\Delta \rho_{\max }=0.27 \mathrm{e} \AA^{-3}$

$\Delta \rho_{\min }=-0.15$ e $\AA^{-3}$ 


\section{Special details}

Experimental. mp $508 \mathrm{~K}$; UV/Vis $\lambda \max (\varepsilon)=243 \mathrm{~nm}\left(12215 M^{-1} \mathrm{~cm}^{-1}\right), 384 \mathrm{~nm}\left(26116 M^{-1} \mathrm{~cm}^{-1}\right)$; IR (neat): $551.84(m)$, $586.34(\mathrm{~m}), 641.18(\mathrm{~m}), 705.16(\mathrm{~m}), 716.43(\mathrm{~m}), 740.14(\mathrm{~m}), 790.77(\mathrm{~m}-\mathrm{s}), 817.7$, (versus $), 838.08(\mathrm{~s}), 863.88(\mathrm{~s}), 937.29$ $(\mathrm{m}), 955.06(\mathrm{~m}), 966.85(\mathrm{~m}), 1014.07(\mathrm{~m}), 1060.05(\mathrm{~m}), 1107.65(\mathrm{~m}), 1166.55(\mathrm{~m}), 1193.28(\mathrm{~m}), 1211.1(\mathrm{~m}), 1238.58(\mathrm{~m})$, $1274.86(\mathrm{~m}), 1295.31(\mathrm{~m}), 1345.37(\mathrm{w}), 1375.47(\mathrm{~m}), 1409.84(\mathrm{~m}-\mathrm{s}), 1456.61(\mathrm{~m}), 1497.28(\mathrm{~s}), 1508.13(\mathrm{~m}), 1526.25(\mathrm{~m})$, 1586.19 (s-versus), $1612.45(\mathrm{~m}), 1636.29$ (w), $1807.98(\mathrm{w}), 1904.79(\mathrm{w}), 2725.8(\mathrm{w}), 2858.33(\mathrm{w}), 2914.98$, (w), 3018.47 (w); ${ }^{1} \mathrm{H}$ NMR $\left(300 \mathrm{MHz}, \mathrm{CDCl}_{3}\right): \delta 8.60(\mathrm{~s}, 2 \mathrm{H}), 7.49(\mathrm{~s}, 2 \mathrm{H}), 7.12(\mathrm{~m}, 8 \mathrm{H}), 2.40(\mathrm{~s}, 6 \mathrm{H}) ;{ }^{13} \mathrm{C} \mathrm{NMR}\left(300 \mathrm{MHz}, \mathrm{CDCl}_{3}\right): \delta$ $151.4258,148.3818,146.3021,136.5105,131.4301,129.9226,129.8156,121.0701,21.0769$

Geometry. All e.s.d.'s (except the e.s.d. in the dihedral angle between two 1.s. planes) are estimated using the full covariance matrix. The cell e.s.d.'s are taken into account individually in the estimation of e.s.d.'s in distances, angles and torsion angles; correlations between e.s.d.'s in cell parameters are only used when they are defined by crystal symmetry. An approximate (isotropic) treatment of cell e.s.d.'s is used for estimating e.s.d.'s involving 1.s. planes.

Fractional atomic coordinates and isotropic or equivalent isotropic displacement parameters $\left(\hat{A}^{2}\right)$

\begin{tabular}{lllll}
\hline & $x$ & $y$ & $z$ & $U_{\text {iso }} * U_{\text {eq }}$ \\
\hline S1 & 1.0000 & $0.43225(6)$ & 0.7500 & $0.04723(16)$ \\
C1 & $0.98232(4)$ & $0.8419(2)$ & $0.7119(2)$ & $0.0554(3)$ \\
H1 & 0.9694 & 0.9702 & 0.6832 & $0.066^{*}$ \\
C2 & $0.96895(4)$ & $0.63105(19)$ & $0.68436(18)$ & $0.0476(3)$ \\
N1 & $0.92443(3)$ & $0.36610(17)$ & $0.59260(16)$ & $0.0488(3)$ \\
C3 & $0.93398(4)$ & $0.56950(19)$ & $0.60827(19)$ & $0.0490(3)$ \\
H3 & 0.9179 & 0.6800 & 0.5696 & $0.059^{*}$ \\
C4 & $0.88949(4)$ & $0.31548(19)$ & $0.52032(16)$ & $0.0449(3)$ \\
C5 & $0.85902(4)$ & $0.4422(2)$ & $0.5468(2)$ & $0.0529(3)$ \\
H5 & 0.8612 & 0.5722 & 0.6126 & $0.063^{*}$ \\
C6 & $0.82570(4)$ & $0.3767(3)$ & $0.4762(2)$ & $0.0582(4)$ \\
H6 & 0.8057 & 0.4645 & 0.4943 & $0.070^{*}$ \\
C7 & $0.82125(4)$ & $0.1822(2)$ & $0.37840(19)$ & $0.0553(3)$ \\
C8 & $0.85167(4)$ & $0.0561(2)$ & $0.35382(19)$ & $0.0540(3)$ \\
H8 & 0.8494 & -0.0745 & 0.2889 & $0.065^{*}$ \\
C9 & $0.88525(4)$ & $0.1194(2)$ & $0.42325(19)$ & $0.0498(3)$ \\
H9 & 0.9052 & 0.0310 & 0.4053 & $0.060^{*}$ \\
C10 & $0.78489(6)$ & $0.1105(4)$ & $0.3019(3)$ & $0.0832(6)$ \\
H10A & 0.7841 & 0.1239 & 0.1756 & $0.125^{*}$ \\
H10B & 0.7666 & 0.2030 & 0.3478 & $0.125^{*}$ \\
H10C & 0.7807 & -0.0411 & 0.3336 & $0.125^{*}$ \\
& & & & \\
\hline
\end{tabular}

Atomic displacement parameters $\left(\AA^{2}\right)$

\begin{tabular}{lllllll}
\hline & $U^{11}$ & $U^{22}$ & $U^{33}$ & $U^{12}$ & $U^{13}$ & $U^{23}$ \\
\hline S1 & $0.0603(3)$ & $0.0289(2)$ & $0.0533(3)$ & 0.000 & $0.0108(2)$ & 0.000 \\
C1 & $0.0585(8)$ & $0.0306(5)$ & $0.0780(9)$ & $0.0020(5)$ & $0.0118(7)$ & $0.0022(5)$ \\
C2 & $0.0570(8)$ & $0.0343(5)$ & $0.0527(7)$ & $0.0002(5)$ & $0.0140(5)$ & $0.0022(5)$ \\
N1 & $0.0557(6)$ & $0.0379(5)$ & $0.0534(6)$ & $-0.0001(4)$ & $0.0082(5)$ & $0.0000(4)$ \\
C3 & $0.0573(8)$ & $0.0368(6)$ & $0.0538(7)$ & $0.0016(5)$ & $0.0115(6)$ & $0.0043(5)$ \\
C4 & $0.0546(7)$ & $0.0352(5)$ & $0.0457(6)$ & $-0.0002(5)$ & $0.0105(5)$ & $0.0030(4)$ \\
C5 & $0.0613(8)$ & $0.0406(6)$ & $0.0579(8)$ & $0.0020(5)$ & $0.0134(6)$ & $-0.0066(5)$ \\
C6 & $0.0553(8)$ & $0.0543(7)$ & $0.0663(9)$ & $0.0067(6)$ & $0.0146(6)$ & $-0.0026(6)$
\end{tabular}


supporting information

\begin{tabular}{lllllll} 
C7 & $0.0586(8)$ & $0.0555(8)$ & $0.0521(7)$ & $-0.0049(6)$ & $0.0075(6)$ & $0.0013(6)$ \\
C8 & $0.0690(9)$ & $0.0418(6)$ & $0.0519(7)$ & $-0.0047(6)$ & $0.0084(6)$ & $-0.0050(5)$ \\
C9 & $0.0601(8)$ & $0.0341(5)$ & $0.0560(7)$ & $0.0033(5)$ & $0.0108(6)$ & $0.0000(5)$ \\
C10 & $0.0664(11)$ & $0.0948(14)$ & $0.0874(13)$ & $-0.0101(10)$ & $-0.0035(10)$ & $-0.0136(10)$ \\
\hline
\end{tabular}

Geometric parameters $\left(A,{ }^{\circ}\right)$

\begin{tabular}{|c|c|c|c|}
\hline $\mathrm{S} 1-\mathrm{C} 2^{\mathrm{i}}$ & $1.7167(13)$ & $\mathrm{C} 5-\mathrm{H} 5$ & 0.9300 \\
\hline $\mathrm{S} 1-\mathrm{C} 2$ & $1.7168(13)$ & $\mathrm{C} 6-\mathrm{C} 7$ & $1.392(2)$ \\
\hline $\mathrm{C} 1-\mathrm{C} 2$ & $1.3762(17)$ & $\mathrm{C} 6-\mathrm{H} 6$ & 0.9300 \\
\hline $\mathrm{C} 1-\mathrm{C} 1^{\mathrm{i}}$ & $1.403(3)$ & $\mathrm{C} 7-\mathrm{C} 8$ & $1.384(2)$ \\
\hline $\mathrm{C} 1-\mathrm{H} 1$ & 0.9300 & $\mathrm{C} 7-\mathrm{C} 10$ & $1.501(2)$ \\
\hline $\mathrm{C} 2-\mathrm{C} 3$ & $1.439(2)$ & $\mathrm{C} 8-\mathrm{C} 9$ & $1.379(2)$ \\
\hline $\mathrm{N} 1-\mathrm{C} 3$ & $1.2802(16)$ & $\mathrm{C} 8-\mathrm{H} 8$ & 0.9300 \\
\hline $\mathrm{N} 1-\mathrm{C} 4$ & $1.4122(18)$ & $\mathrm{C} 9-\mathrm{H} 9$ & 0.9300 \\
\hline $\mathrm{C} 3-\mathrm{H} 3$ & 0.9300 & $\mathrm{C} 10-\mathrm{H} 10 \mathrm{~A}$ & 0.9600 \\
\hline $\mathrm{C} 4-\mathrm{C} 5$ & $1.3907(19)$ & $\mathrm{C} 10-\mathrm{H} 10 \mathrm{~B}$ & 0.9600 \\
\hline $\mathrm{C} 4-\mathrm{C} 9$ & $1.3963(17)$ & $\mathrm{C} 10-\mathrm{H} 10 \mathrm{C}$ & 0.9600 \\
\hline $\mathrm{C} 5-\mathrm{C} 6$ & $1.377(2)$ & & \\
\hline $\mathrm{C} 2 \mathrm{i}-\mathrm{S} 1-\mathrm{C} 2$ & $91.43(9)$ & $\mathrm{C} 5-\mathrm{C} 6-\mathrm{H} 6$ & 119.2 \\
\hline $\mathrm{C} 2-\mathrm{C} 1-\mathrm{C}^{\mathrm{i}}$ & $112.53(9)$ & $\mathrm{C} 7-\mathrm{C} 6-\mathrm{H} 6$ & 119.2 \\
\hline $\mathrm{C} 2-\mathrm{C} 1-\mathrm{H} 1$ & 123.7 & $\mathrm{C} 8-\mathrm{C} 7-\mathrm{C} 6$ & 117.54 (14) \\
\hline $\mathrm{C} 1 \mathrm{i}-\mathrm{C} 1-\mathrm{H} 1$ & 123.7 & $\mathrm{C} 8-\mathrm{C} 7-\mathrm{C} 10$ & $120.90(15)$ \\
\hline $\mathrm{C} 1-\mathrm{C} 2-\mathrm{C} 3$ & $127.48(12)$ & $\mathrm{C} 6-\mathrm{C} 7-\mathrm{C} 10$ & $121.56(15)$ \\
\hline $\mathrm{C} 1-\mathrm{C} 2-\mathrm{S} 1$ & $111.75(11)$ & $\mathrm{C} 9-\mathrm{C} 8-\mathrm{C} 7$ & $121.63(12)$ \\
\hline $\mathrm{C} 3-\mathrm{C} 2-\mathrm{S} 1$ & $120.76(9)$ & $\mathrm{C} 9-\mathrm{C} 8-\mathrm{H} 8$ & 119.2 \\
\hline $\mathrm{C} 3-\mathrm{N} 1-\mathrm{C} 4$ & $119.09(12)$ & $\mathrm{C} 7-\mathrm{C} 8-\mathrm{H} 8$ & 119.2 \\
\hline $\mathrm{N} 1-\mathrm{C} 3-\mathrm{C} 2$ & $121.53(12)$ & $\mathrm{C} 8-\mathrm{C} 9-\mathrm{C} 4$ & $120.43(13)$ \\
\hline $\mathrm{N} 1-\mathrm{C} 3-\mathrm{H} 3$ & 119.2 & $\mathrm{C} 8-\mathrm{C} 9-\mathrm{H} 9$ & 119.8 \\
\hline $\mathrm{C} 2-\mathrm{C} 3-\mathrm{H} 3$ & 119.2 & $\mathrm{C} 4-\mathrm{C} 9-\mathrm{H} 9$ & 119.8 \\
\hline $\mathrm{C} 5-\mathrm{C} 4-\mathrm{C} 9$ & $118.30(13)$ & $\mathrm{C} 7-\mathrm{C} 10-\mathrm{H} 10 \mathrm{~A}$ & 109.5 \\
\hline $\mathrm{C} 5-\mathrm{C} 4-\mathrm{N} 1$ & $124.28(11)$ & $\mathrm{C} 7-\mathrm{C} 10-\mathrm{H} 10 \mathrm{~B}$ & 109.5 \\
\hline $\mathrm{C} 9-\mathrm{C} 4-\mathrm{N} 1$ & $117.35(12)$ & $\mathrm{H} 10 \mathrm{~A}-\mathrm{C} 10-\mathrm{H} 10 \mathrm{~B}$ & 109.5 \\
\hline $\mathrm{C} 6-\mathrm{C} 5-\mathrm{C} 4$ & $120.49(12)$ & $\mathrm{C} 7-\mathrm{C} 10-\mathrm{H} 10 \mathrm{C}$ & 109.5 \\
\hline $\mathrm{C} 6-\mathrm{C} 5-\mathrm{H} 5$ & 119.8 & $\mathrm{H} 10 \mathrm{~A}-\mathrm{C} 10-\mathrm{H} 10 \mathrm{C}$ & 109.5 \\
\hline $\mathrm{C} 4-\mathrm{C} 5-\mathrm{H} 5$ & 119.8 & $\mathrm{H} 10 \mathrm{~B}-\mathrm{C} 10-\mathrm{H} 10 \mathrm{C}$ & 109.5 \\
\hline $\mathrm{C} 5-\mathrm{C} 6-\mathrm{C} 7$ & $121.62(14)$ & & \\
\hline $\mathrm{C} 1-\mathrm{C} 1-\mathrm{C} 2-\mathrm{C} 3$ & $-179.60(16)$ & $\mathrm{N} 1-\mathrm{C} 4-\mathrm{C} 5-\mathrm{C} 6$ & $-177.78(13)$ \\
\hline $\mathrm{C} 1-\mathrm{C} 1-\mathrm{C} 2-\mathrm{S} 1$ & $-0.6(2)$ & $\mathrm{C} 4-\mathrm{C} 5-\mathrm{C} 6-\mathrm{C} 7$ & $0.7(2)$ \\
\hline $\mathrm{C} 2 \mathrm{i}-\mathrm{S} 1-\mathrm{C} 2-\mathrm{C} 1$ & $0.23(8)$ & $\mathrm{C} 5-\mathrm{C} 6-\mathrm{C} 7-\mathrm{C} 8$ & $-0.2(2)$ \\
\hline $\mathrm{C} 2 \mathrm{i}-\mathrm{S} 1-\mathrm{C} 2-\mathrm{C} 3$ & $179.28(15)$ & $\mathrm{C} 5-\mathrm{C} 6-\mathrm{C} 7-\mathrm{C} 10$ & $179.82(17)$ \\
\hline $\mathrm{C} 4-\mathrm{N} 1-\mathrm{C} 3-\mathrm{C} 2$ & $178.67(12)$ & $\mathrm{C} 6-\mathrm{C} 7-\mathrm{C} 8-\mathrm{C} 9$ & $0.1(2)$ \\
\hline $\mathrm{C} 1-\mathrm{C} 2-\mathrm{C} 3-\mathrm{N} 1$ & $-178.68(14)$ & $\mathrm{C} 10-\mathrm{C} 7-\mathrm{C} 8-\mathrm{C} 9$ & $-179.95(16)$ \\
\hline $\mathrm{S} 1-\mathrm{C} 2-\mathrm{C} 3-\mathrm{N} 1$ & $2.4(2)$ & $\mathrm{C} 7-\mathrm{C} 8-\mathrm{C} 9-\mathrm{C} 4$ & $-0.4(2)$ \\
\hline $\mathrm{C} 3-\mathrm{N} 1-\mathrm{C} 4-\mathrm{C} 5$ & $-35.2(2)$ & $\mathrm{C} 5-\mathrm{C} 4-\mathrm{C} 9-\mathrm{C} 8$ & $0.9(2)$ \\
\hline
\end{tabular}


$\mathrm{C} 3-\mathrm{N} 1-\mathrm{C} 4-\mathrm{C} 9 \quad 148.02(13)$

$\mathrm{C} 9-\mathrm{C} 4-\mathrm{C} 5-\mathrm{C} 6$

$-1.0(2)$

$\mathrm{N} 1-\mathrm{C} 4-\mathrm{C} 9-\mathrm{C} 8$

$177.88(12)$

Symmetry code: (i) $-x+2, y,-z+3 / 2$. 